of neuroleptic treatment. Examination of the subjects shows that actually, those with TD have twofold more psychiatric admissions compared with those without TD; thus they are probably the more severely ill group of patients. It is to be expected that they might have received higher neuroleptic dosage during hospital stays, and so the cumulative neuroleptic dosage might have been higher in the group with TD. The lower current neuroleptic dosage in the group with TD compared with those without TD may reflect the attending doctors' attempts to reduce dosage of neuroleptics after onset of TD, rather than these patients requiring lower maintenance dose of neuroleptics. To establish that lithium exposure is really a risk factor for the development of TD, the cumulative neuroleptic dosage should be matched for the two groups of patients.

Department of Psychiatry

Helen Chiu

Chinese University

Prince of Wales Hospital

Shatin, Hong Kong

SIR: Dr Falvey is clearly correct in pointing out the multivariate aetiology of TD. The disease process, ageing and psychotropic medication probably all interact to produce the disorder. Our study, in attempting to elucidate the role of lithium in the development of TD in bipolar affective disorder, is in no way suggesting a univariate iatrogenic model for TD. The literature to date does not support such a model, and such thinking has considerable medico-legal significance.

It is possible, as Dr Chiu argues, that the lower current neuroleptic dosage in the group with TD may be an attempt by the clinician to reduce the dosage of neuroleptics after the onset of TD. A review of the patient records in general does not support such a view. In the majority of patients, the presence of even moderately severe orofacial dyskinesia was not noted by the attending clinician.

The fact that all patients fulfilled Schooler \& Kane research diagnostic criteria for TD meant that each had at least 2 months' neuroleptic exposure. We agree with Dr Volavka that a study of patients treated with lithium but having no previous neuroleptic exposure would be useful. Such patients are, however, few and far between.

Timothy G. DinaN DORA KOHEN

St James's Hospital

James's Street

P.O. Box 580

Dublin 8

\section{The opiate prescribing debate}

SIR: Your readers will have been mislead by a recent letter from Dr John Marks (Journal, October 1989. $155,566)$ in which he suggests that making opiate drugs more available reduces the problems associated with drugs. Dr Marks cites two references and suggests that these support his assertion.

Unfortunately these references are not to clinical trials or studies, but correspondence in which he restates his well-known views. The relationship between prescribing policies and the behaviour of drug users is far from understood. The notion that more liberal prescribing policies will reduce the crime rate remains an important hypothesis which requires testing.

\author{
St George's Hospital Medical School \\ Department of Psychiatry \\ Cranmer Terrace \\ London SW17 ORE
}

ANDREW JOHNS

\section{The evaluation of mental health care systems}

SIR: Häfner \& an der Heiden (Journal, July 1989, 155, 12-17) discuss the evaluation of mental health care systems. They leave a number of questions unanswered and, indeed, unasked.

The question is set as to whether out-patient care affected either the length of time spent in the community or the length of time during readmission to hospital. There is inadequate description of the nature of this care, and no real mention of the alternatives, this despite the earlier plea that "interventions subject to evaluation must be described precisely".

It is debatable whether the two effectiveness criteria cited constitute a valid therapeutic outcome when used in such an unqualified way: perhaps the reasons for readmission would shed some light on how out-patient care is provided so cheaply in this case? Similarly, Fig. 2 of the paper raises the question as to why patients with the highest chance of readmission had the lowest frequency of out-patient contact: surely it is wrong to conclude that frequency of out-patient contact directly influences the chance of readmission, despite allowing for a few of the possible intervening variables (symptoms, length of previous in-patient stay, and living conditions)?

Our main comments are reserved for the method of economic analysis (itself at odds with the request not to overvalue economic factors). Direct monetary cost is used as the sole indicator of total cost and, subsequently, an attempt is made to relate this to (unmeasured) non-monetary costs such as burden of 